\title{
INTEGRACIÓN FINANCIERA EUROPEA El Impacto del Euro en las PYMES
}

Dra. BEATRIZ HERRERA GARCIA (*)

MBA. SORAYA CASTRO HERRERA (**)

\section{INTRODUCCIÓN}

La creación de la Unión Monetaria Europea (UME) y la introducción del Euro constituyen un acontecimiento histórico que, sin duda, tendrá repercusiones de grandes magnitudes para el orden económico mundial. A pesar de ello, el estudio de sus implicaciones ha adoptado, básicamente, una perspectiva regional al ocuparse sobre todo de los efectos de la Unión Monetaria en los propios países integrantes del área. Más aún, los estudios se han centrado en evaluar el impacto en los diversos sectores económicos de cada país.

El presente trabajo está orientado, específicamente, al sector denominado PYMES, pequeñas y medianas empresas que en los últimos años constituyen la columna vertebral de la economía en España y en la Unión Europea, a pesar de la presión creciente de la competencia, la globalización de los mercados, el auge de las fusiones y alianzas estratégicas.

Según cifras estimadas, las PYMES alcanzan el $90 \%$ del conjunto de las empresas; representan más del $60 \%$ del empleo total y tienen aproximadamente el $65 \%$ del volumen de negocios de la Unión Europea (UE) ${ }^{1}$. Lo que las ubica como una importante fuente generadora de nuevos puestos de trabajo y forjadora del desarrollo regional.

Actualmente, no se han realizado estudios puntuales sobre el número de estas empresas, ni del tamaño, estructura y recursos humanos, como grupo específico del conjunto de PYMES. Existe la necesidad de conocer el grado de internacionalización y europeización de estas empresas.

En este estudio se plantea la problemática que sufren las PYMES españolas para integrarse al nuevo sistema económico de integración europea. En el capítulo primero se hace un análisis situacional de las PYMES, basado en el entorno macroeconómico pre y postintegración monetaria.

En el capítulo segundo se identifican las principales ventajas y desventajas por las cuales atraviesan las PYMES en su adecuación al sistema Euro. En el capítulo tercero

(*) Decana y Directora del Instituto de Investigación de Ciencias Financieras y Contables de la Facultad de Ciencias Contables y Miembro del Comité Ejecutivo del Consejo Superior de Investigaciones de la UNMSM.

$\left.{ }^{* *}\right)$ Doctoranda de la Universidad Autónoma de Madrid, España.

1. José Manuel Serra Peris. "La PYME Industrial en el Nuevo Modelo de Crecimiento". En: Economia Industrial $N^{\circ} 310,1996$. José Manuel Serra P. Se desempeñaba como Secretario General Técnico en el Ministerio de Industrial y Energia. 
se determinan las principales acciones y actividades de las entidades reguladoras como son la Comisión Europea, la Comunidad de Madrid y las Cámaras de Comercio e Industria en apoyo a las PYMES $\mathrm{y}$, finalmente, expresamos nuestras conclusiones y recomendaciones.

\section{ENTORNO ECONÓMICO E INSTITUCIONAL Y LOS CRITERIOS QUE.DETERMINAN LA ENTRADA AL EURO DE LAS PYMES ESPAÑOLAS}

\subsection{Política orientada al crecimiento}

Desde décadas pasadas en las economías europeas existió un creciente desfase entre la capacidad de producción y la demanda agregada, por lo que la integración europea exigía un nuevo modelo de crecimiento asentado en los sectores de elevado valor añadido y de tecnologías de vanguardia. Este nuevo modelo de crecimiento combinó la explotación sistemática de las competencias tecnológicas, organizativas, comerciales, etc.; puntos importantes en el desarrollo económico.

La consolidación de esta nueva política de crecimiento conllevó a una reorganización de las grandes empresas que en un principio redujeron su tamaño y/o -en algunos casos radicales-, cambiaron de actividad. Muchas otras optaron por la subcontratación de sus áreas de producción, comercialización, administración, etc., a empresas independientes $o$ asociadas. Es en este momento cuando surge un lazo estrecho entre las grandes empresas y las PYMES. Estas últimas establecieron diversas formas de coopera- ración y alianzas estratégicas para el desarrollo económico, reducción de costos, financiación, etc., todo ello debido a que poseen ventajas como la flexibilidad, la especialización, una estructura organizativa sencilla y poco formalizada, entre otras características.

En esta nueva coyuntura empresarial, en donde lidera una política de subcontratación, las PYMES han tomado fuerza y forman parte importante del aparato económico de España. Algunos autores ${ }^{2}$ sostienen que la participación de las PYMES -sobre todo industriales-, en el desarrollo económico tienden a aumentar y no a disminuir. Esta sentencia es discutible dado que la actual tendencia económica es hacia la fusión y creación de consorcios cuyo principal fin es la ampliación del mercado.

\subsection{Política Macroeconómica}

La política monetaria de estabilidad debe garantizar que el crecimiento de la economía esté acompañado por una expansión de la demanda. Para lograr dicho objetivo se debe dar una reducción progresiva de los déficit presupuestarios, la reducción de los tipos de interés y el control de la inflación.

En 1997, fase inicial del programa de adaptación al Euro (véase Anexo Nos. I y II), el Gobierno español elaboró un Programa de Estabilidad ${ }^{3}$ que publicó en abril de ese mismo año, en donde recoge las previsiones y los compromisos sobre la evolución de las grandes cifras de la economía en el período 1997 - 2000, a fin de garantizar un desarrollo estable. (véase

2. J. M. Serra Peris. En la PYME Industrial y el Nuevo Modelo de Crecimiento.

3. El texto íntegro del "Programa de Convergencia de España 1997", puede verse en Expansión, 11 de abril de 1997. 
Cuadro $\mathrm{N}^{\circ} 1$ ). La decisión del Ejecutivo era de avanzar en la vía de la convergencia con el resto de las economías comunitarias que aspiraban a la moneda única; e igualmente, el compromiso de mantener una política de rigor tras superar el examen de acceso al euro.

En el referido documento prevé un crecimiento del PBI (Producto Bruto Interno) del $3,2 \%$ promedio durante los 4 años. Con una tasa de inflación que fluctué el 2,3\% medido como deflactor del consumo privado. Una balanza comercial positiva del $0,4 \%$ en promedio y un crecimiento del consumo privado del orden del $2,8 \%$ versus un crecimiento del consumo nacional del $0,7 \%$. Por otro lado, se planteó un incremento del empleo que promedia el $2,1 \%$.

Junto con la corrección paulatina del déficit y la inflación, en el programa se predice una evolución favorable de la deuda pública, que del 68,2 por 100 del PIB a fi-

CUADRO N 1

PROGRAMA DE CONVERGENCIA (1997-2000)

Presupuestado

\begin{tabular}{|c|c|c|c|c|c|}
\hline CONCEPTOS & 1997 & 1998 & 1999 & 2000 & MEDIA \\
\hline \multicolumn{6}{|l|}{ PBI Y AGREGADOS (\% variación real) } \\
\hline Consumo privado nacional & 2,7 & 2,7 & 2,8 & 3,0 & 2,8 \\
\hline Consumo público & $-0,3$ & 1,0 & 1,0 & 1,0 & 0,7 \\
\hline Formación bruta del capital fijo & 4,0 & 7,1 & 7,8 & 5,6 & 6,1 \\
\hline Demanda nacional & 2,5 & 3,4 & 3,7 & 3,3 & 3,2 \\
\hline Exportación de bienes y servicios & 9,9 & 9,5 & 9,7 & 8,8 & 9,5 \\
\hline Importación de bienes y servicios & 8,1 & 9,6 & 10,0 & 8,9 & 9,1 \\
\hline Saldo exterior (contribución al crecimiento del PBI) & 0,4 & $-0,2$ & $-0,3$ & $-0,2$ & $\mathbf{0 , 1}$ \\
\hline PBI & 3,0 & 3,2 & 3,4 & 3,2 & 3,2 \\
\hline \multicolumn{6}{|l|}{ OTRAS VARIABLES } \\
\hline Deflactor del consumo privado (var.\%) & 2,5 & 2,3 & 2,3 & 2,2 & 2,3 \\
\hline Empleo (var. \%) & 2,0 & 2,2 & 2,2 & 2,1 & 2,1 \\
\hline Cap. $(+)$ nec. (-) finan. resto del mundo (\% PBI) & 1,9 & 1,7 & 1,5 & 1,5 & $\mathbf{1 , 6}$ \\
\hline \multicolumn{6}{|l|}{ ESCENARIO DE CRECIMIENTO ELEVADO } \\
\hline PBI (\% variación real) & 3,2 & 3,5 & 3,8 & 3,4 & 3,5 \\
\hline Deflactor del consumo privado & 2,5 & 2,6 & 2,8 & 2,6 & 2,6 \\
\hline Déficit AA.PP./PBI & 3,0 & $-2,3$ & $-1,7$ & $-1,3$ & ----- \\
\hline Deuda/PBI & 68,0 & 67,1 & 65,4 & 63,5 & $-\cdots$ \\
\hline \multicolumn{6}{|l|}{ ESCENARIO DE CRECIMIENTO MODERADO } \\
\hline PBI (\% variación real) & 2,5 & 2,6 & 2,8 & 2,6 & 2,6 \\
\hline Deflactor del consumo privado & 2,4 & 2,1 & 2,0 & 2,0 & 2,1 \\
\hline Déficit AA.PP/PBI & $-3,0$ & $-2,7$ & $-2,5$ & $-2,4$ & ----- \\
\hline Deuda/PBI & 68,7 & 68,6 & 68,5 & 68,3 & ---- \\
\hline
\end{tabular}

Fuente: Programa de Convergencia

Elaboración: Propia 
nales de 1997 , se reducirá al $65,3 \%$ por 100 en el 2000.

Según cifras de los principales indicadores económicos extraídos de los boletines estadísticos del Banco de España y del Ministerio de Economía y Hacienda (a través del Tesoro Público), el consumo tanto privado (hogares) como público ha superado sustancialmente las cifras proyectadas por el Gobierno en el Programa de Estabilidad.

Asimismo, la brecha existente entre las importaciones y exportaciones de bienes y servicios se ha ampliado debido principalmente a las importaciones de servicios registrados entre 1998 y 1999 . La formación bruta del capital fijo se redujo en 100 puntos básicos; sin embargo, se mantiene por encima de las cifras proyectadas para el mismo periodo en el Programa de Estabilidad.
El PBI superó satisfactoriamente los índices estimados lo cual lo ubica por encima de la media de la UE. Este indicador se situó en $4 \%, 3,7 \%$ entre 1998 y 1999 , respectivamente.

Con respecto a las variables sociales se observa un incremento satisfactorio de la tasa de empleo que alcanzó niveles de 3,7\% y $3,4 \%$ entre 1998 y 1999 versus los estimados que se proyectaron en $2,2 \%$ en el mismo período. La inflación medida como el deflactor del consumo privado mantuvo -en promedio-, el estimado en el mencionado Programa de Estabilidad (2,0\% y $2,8 \%$ ).

En términos generales, se observa un crecimiento económico sustentado básicamente en el incremento del consumo final tanto de las administraciones públicas como de los hogares, lo que ha implicado elevar las importaciones. El mayor consumo estaría sustentado en el aumento de puestos de trabajo, principalmente de la rama de los asalariados.

\section{CUADRO No 2}

CONTABILIDAD NACIONAL (1998-2000)

\begin{tabular}{|l|r|r|r|}
\hline \multicolumn{1}{|c|}{ CONCEPTOS } & $\mathbf{1 9 9 8}$ & $\mathbf{1 9 9 9}$ & $\mathbf{2 0 0 0 ^ { * * }}$ \\
\hline PBI Y AGREGADOS (\% variación real)* & & & \\
\hline Consumo privado nacional & 4,1 & 4,4 & 3,7 \\
Consumo público & 2,0 & 1,8 & 1,2 \\
Formación bruta del capital fijo & 9,2 & 8,3 & 9,2 \\
Demanda nacional & 5,0 & 4,9 & 4,6 \\
Exportación de bienes y servicios & 7,1 & 8,5 & 9,3 \\
Importación de bienes y servicios & 11,1 & 12,6 & 11,9 \\
Saldo exterior (contribución al crecimiento del PBI) & $-1,0$ & $-1,2$ & $-0,9$ \\
PBI & 4,0 & 3,7 & 3,7 \\
\hline OTRAS VARIABLES & & & \\
\hline Deflactor del consumo privado (var.\%) & 2,0 & 2,8 & 2,7 \\
Empleo (var.\%) & 3,7 & 3,4 & 2,0 \\
\hline
\end{tabular}

(*) Datos corregidos de efectos estacionales y de calendario.

(**) Previsiones del Gobierno.

Fuente: Banco de España y Tesoro Público.

Elaboración: Propia 
Por su parte, las PYMES abarcan casi el $90 \%$ del total de empresas en España y mantienen un promedio de 310 mil trabajadores en sus planillas según consta en las bases de datos del Banco de España y publicado en sus estadísticas anuales. (véase Cuadro $\mathrm{N}^{\circ} 3$ ). el valor añadido bruto al coste de factores ha tenido una tendencia inconstante registrando un notable descenso a partir de 1996 del orden del $15 \%$ para las referidas medianas empresas motivado probablemente por el incremento en los precios de los insumos o los productos primarios y por

\section{CUADRO $\mathbf{N}^{\circ} 3$}

CARACTERÍSTICAS GENERALES DE LAS PYMES

Año 1997

\begin{tabular}{|l|c|c|}
\hline DESCRIPCIÓN & PEQUEÑAS & MEDIANAS \\
\hline Número de empresas (\%) & 56,3 & 30,6 \\
\hline Trabajadores * & 86,5 & 220,7 \\
\hline Fijos & 62,5 & 157,6 \\
No Fijos & 23,9 & 63,1 \\
\hline Valor Añadido bruto al coste de factores (\%) & 3,7 & 11,7 \\
\hline
\end{tabular}

* Número medio de trabajadores durante el año, expresado en miles.

Fuente: Banco de España

El valor añadido bruto de estas empresas totalizó en 1997 en 3,7\% para lo que se refiere pequeñas empresas y un

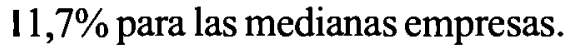

Al respecto, se puede observar (Gráfico $\mathrm{N}^{\circ}$ 1) que a lo largo de la década pasada el aumento del consumo de explotación. La tendencia del valor añadido bruto al coste de los factores para el caso de las pequeñas empresas se ha mantenido relativamente estable a pesar de registrar entre 1997 y 1998 una caída del $16 \%$.

\section{GRÁFICO No 1}

VALOR AÑADIDO AL COSTE DE FACTORES

(1989 - 1998)

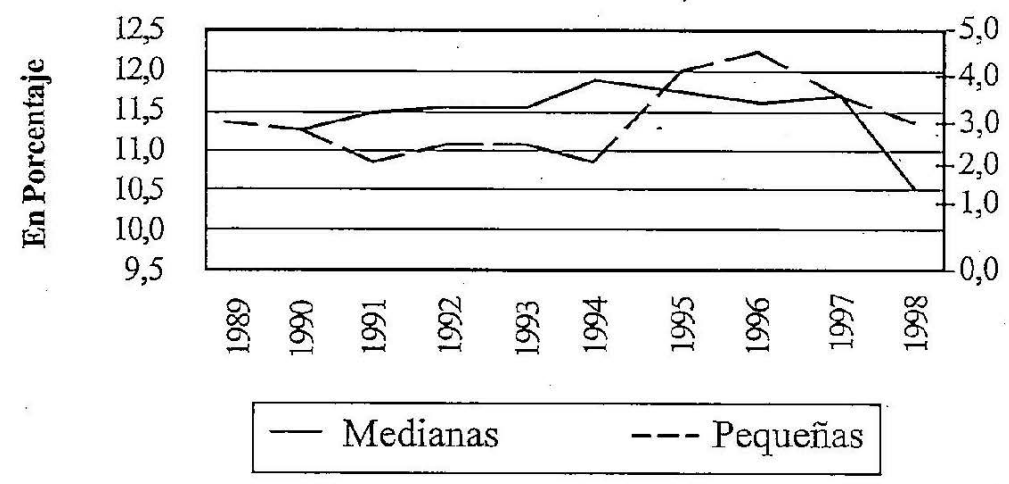

Fuente: Banco de España. 
A pesar de registrar menores utilidades sobre el coste de sus factores y, por consiguiente, sobre las utilidades netas son más las empresas que han venido realizando inversiones. Estadísticas del Banco de España arrojan que de un $38 \%$ a un $43 \%$ de las empresas registran inversiones en inmovilizado material significativa, definida como superior al $10 \%$ del stock de inmo-vilizado material al inicio del ejercicio. Lo que nos sugeriría que las PYMES se preparan para la conversión. territorio español. Asimismo, las convierte en la principal fuente de creación de nuevos empleos.

\subsection{Políticas de reformas estructurales}

En este punto aún se mantienen pendientes, por parte del gobierno, el establecer condiciones para que el funcionamiento de los mercados de productos y servicios, y de factores, sean transparentes, eficaces y competitivos. Ello conllevaría a la necesaria

\section{CUADRO N 4}

INVERSIÓN DE LAS PEQUEÑAS EMPRESAS(*)

(1990 - 1998)

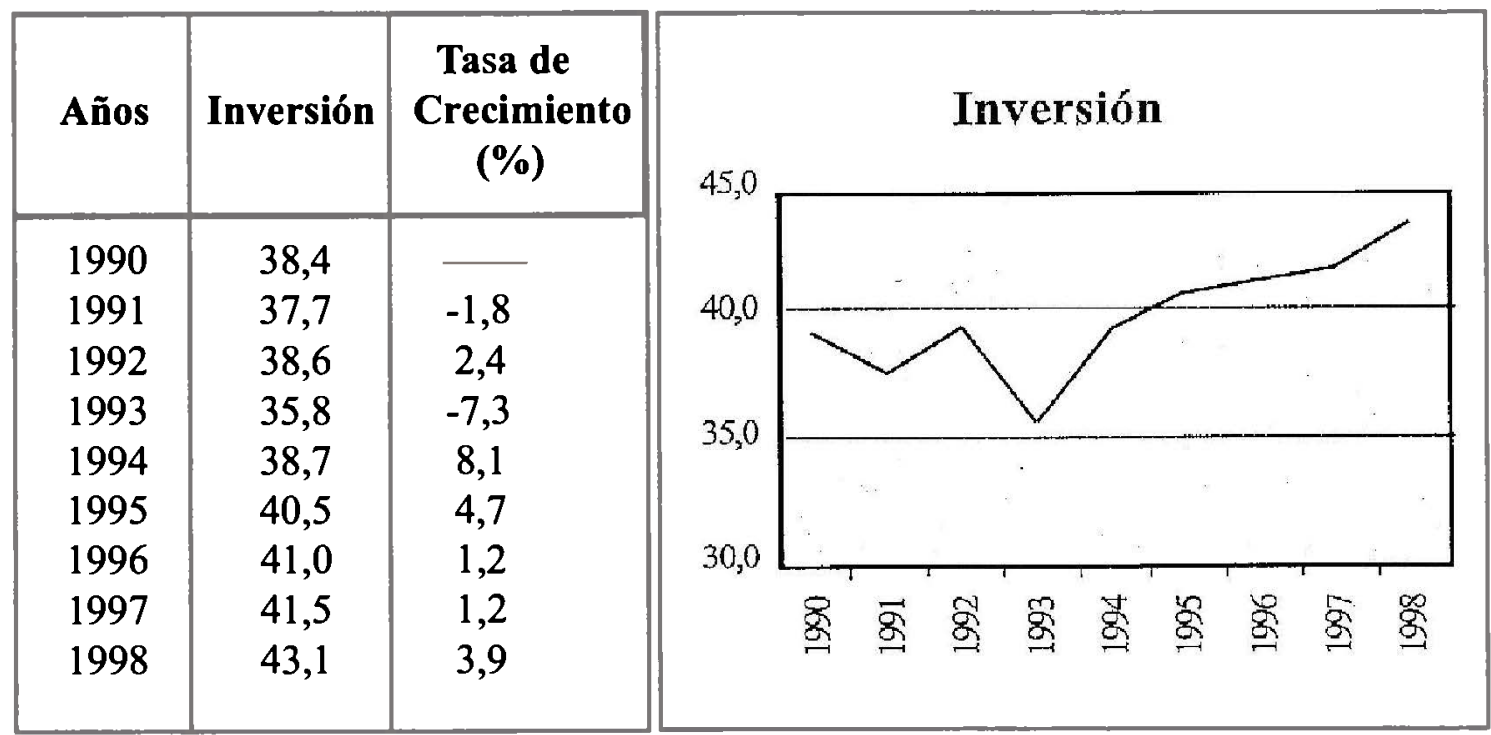

(*) Porcentaje de empresas con formación bruta de capital fijo significativa respecto del total de la muestra. Porcentaje de empresas con inversión en inmovilizado material significativo, definido como superior al $10 \%$ del stock de inmovilizado material al inicio del ejercicio.

Fuente: Banco de España.

Este marco coyuntural económico favorable ha motivado que el peso de las PYMES dentro del aparato productivo español se incremente sustancialmente, llevando a estas empresas -pequeñas y medianas- a representar más del $90 \%{ }^{4} \mathrm{del}$ total de empresas que operan dentro del aplicación de medidas estructurales relacionadas con la liberalización y regulación de mercados, la consolidación de la competencia en sectores monopolísticos, la modernización de los poderes públicos, la simplificación legal y administrativa.

4. Estadísticas del Banco de España (véase Cuadro $N^{\circ} 3$ ). 
Asimismo, es preciso mejorar el entorno financiero de las empresas sobre todo de las PYMES que padecen de problemas sistemáticos de financiación debido, básicamente, a la dificultad de acceso a préstamos bancarios con tipos de interés razonables; así como mejorar las exigencias en garantías, desarrollar instrumentos financieros específicos.

Finalmente, en política de reformas estructurales, el Gobierno debe adaptar las instituciones del mercado de trabajo aumentando su transparencia y combinando medidas que apoyen la movilidad funcional y geográfica; así como la formación y orientación profesional. Fomentar iniciativas de desarrollo y empleo.

\subsection{Criterios que determinan que las empresas asuman el Euro como moneda única}

A pesar de que oficialmente el Euro ha entrado en funcionamiento (electrónico) desde el $1^{\circ}$ de enero de 1999, las PYMES españolas no sienten - del todo-, el peso de operar bajo el sistema Euro. Sólo las grandes empresas utilizan esta moneda en operaciones financieras de gran magnitud.

Oficialmente, a partir del $1^{\circ}$ de enero del 2002 dejará de existir la dualidad de las monedas, y existirá en el mercado sólo el Euro como única moneda de curso legal; por lo que las pequeñas y microempresas cuentan con 2 años aproximadamente para determinar y evaluar criterios que definan su ingreso exitoso en esta nueva coyuntura. A continuación mencionaremos los que a nuestro parecer serían los más relevantes:

El primer factor a tener en cuenta es su relación con el cliente final y de proveedores principales. Las PYMES deberán implantar políticas de operatividad en función de estos agentes. Según el análisis Ford, las empresas mantienen debilidades o fortalezas dependiendo de su relación con los clientes, proveedores, competidores directos (misma rama de la industria), los competidores indirectos (productos sustitutos). Asimismo, se debe tomar en cuenta la presión de los socios. En base a estas relaciones las PYMES asumirán más rápidamente el Euro o retrazarán su operatividad con la moneda única europea.

Asimismo, una vez que se abra el mercado, las relaciones con estos elementos económicos se volverán más competitivas y productivas lo que redundará en una mayor eficiencia en sus sistemas de costos y presupuestos, criterio muy importante para iniciar una carrera satisfactoria frente al resto de las PYMES de Europa.

La entrada al Euro implica que las empresas se adapten rápidamente a la nueva coyuntura del mercado, donde será muy importante la característica fundamental de las PYMES: la flexibilidad. Por lo tanto, gozan de la capacidad de adecuarse velozmente y de implantar mecanismos de reformas en sus diferentes áreas, inclusive gestionar un cambio de actividad.

En una encuesta realizada en 1997 por Andersen Consulting sobre la opinión de las empresas al cambio peseta/euro y sus implicancias, resultó que el $75 \%$ de las encuestadas pensó que el cambio sería beneficioso para sus negocios, con rebajas de los costes financieros, estimulación del crecimiento económico con tipos de interés más bajos y una inflación menor. Sin embargo, temían a los costes de la conversión y a perder al tipo cambio como elemento de ajuste.

No cabe duda que la apreciación de las empresas encuestadas era acertada. La Unión Monetaria ha llevado a las economías de los países miembros hacia la convergencia 
lo que ha ocasionado la reducción de los costes financieros, crecimiento económico con tasas de inflación menores respecto de años anteriores. Sin embargo, aún quedan dos años de dualidad monetaria y los efectos finales de la última fase (a partir del $1^{\circ}$ de julio del 2002 - (véase Anexo II). De la gestión de las dos fases anteriores dependerá la competitividad de la empresa.

\section{IMPACTO DEL EURO EN LAS PYMES ESPAÑOLAS}

Basada en una experiencia empírica, algunas PYMES aún no han evaluado las posibles ventajas y consecuencias de su incorporación a la moneda única. Además, como no han previsto un programa de adecuación a la nueva coyuntura.

El efecto Euro influirá en las empresas en todas sus actividades y áreas. El costo del impacto del cambio dependerá de cierto modo del sector, tamaño y localización de las PYMES, (véase Anexo III). En las siguientes líneas plantearemos las ventajas generales y las posibles desventajas que sufrirían las PYMES españolas luego de entrar en vigencia definitivamente el Euro como única moneda de curso legal.

\subsection{Ventajas generales para las PYMES españolas}

Las principales ventajas de la integración monetaria/Euro están orientadas básicamente al área financiero.

Uno de los beneficios principales del ingreso al sistema Euro es la estabilidad cambiaria. Al existir una única moneda el riesgo en el tipo de cambio (volatilidad de la moneda) se reduce. Por lo tanto, disminuye cuantiosamente el costo de cobertura de riesgos. Asimismo, se reducen los costos de transacción que redundará en un precio menor y más competitivo.
Los tipos de interés se reducirán abriendo campo a futuros financiamientos baratos, de preferencia de largo plazo. $\mathrm{Al}$ integrarse la economía, los tipos de interés caminarán hacia la convergencia; luego del período transitorio, lo único que diferenciará de un país a otro serán las primas de riesgo.

La competencia en el sector financiero no sólo traerá bajas tasas de interés, sino nuevas formas de financiación, mayor oferta de productos de inversión y nuevas formas de pago.

\subsection{Perturbaciones del Euro en las empresas españolas}

Los costos que implican la convertibilidad en todas las áreas de las empresas: administrativas, contables, financieras, de información, de sistemas, etc., que por la estructura de sus negocios, las PYMES tienen que afrontar son altos. La falta de medios (a diferencia de las grandes empresas), se convierte en el principal inconveniente del cambio al nuevo sistema monetario.

Al respecto, cabe indicar que las PYMES por su estructura financian sus inversiones a través de la generación interna de recursos; el cambio estructural implicará inversiones extras que llevarán a estas empresas a acudir a intermediarios financieros con altos tipos de interés. Es en este punto donde el sector público.juega un papel importante que más adelante señalaremos.

Al incorporarse el Euro, uno de los principales inconvenientes está referido al aprendizaje y establecimiento de los nuevos precios, la convertibilidad peseta/euro (redondeos y decimales), no resulta del todo agradable cuando no se tratan de números múltiplos de 5 ó 10 (1 Euro $=166,866$ pesetas). Cabe señalar que en esta etapa de 
dualidad, las empresas que se vienen convirtiendo al Euro han tenido que implantar en sus sistemas (cajas registradoras, principalmente), el doble precio (precio en pesetas y el equivalente en Euros), generando un costo adicional a sus sistemas de adecuación; sin embargo, estas empresas son en su mayoría grandes y medianas empresas.

Por otro lado, la competencia va a influir en las PYMES y los costos serán parte clave de la competitividad. Las empresas tendrán que reducir los costes que sean superfluos y racionalizar de transacciones, a través de un nuevo sistema de control de costos y presupuestos.

Actualmente, las PYMES que trabajan con la dualidad de las monedas tuvieron que adecuar sus aplicaciones y modificar su sistema contable. Las PYMES que aún no se han incorporado a ese nuevo modelo, eventualmente, tendrían que esperar al 2002; aprender de la experiencia de sus similares y enfrentarse a los problemas que pueda traer su ingreso tardío al sistema.

En términos generales, con la moneda única desaparecerán los riesgos del tipo de cambio, lo que influirá favorablemente en la reducción de los tipos de interés y que a su vez impulsará el comercio y a la inversión. Se incrementará el endeudamiento de largo plazo.

Los costes de la transacción de cambio durante las operaciones comerciales y algunos costes de gestión (por ejemplo flujos de efectivo "cash flow") desaparecerán. Además, al disminuir los costes internos de gestión de cambios es posible obtener una mayor rentabilidad de las inversiones.

La principal desventaja de la implementación de la moneda única serán los costes temporales que se generarán por el cambio que son el establecimiento de nuevos precios, la adaptación de tecnologías y la adaptación de sistemas.

\section{ACTIVIDADES EN FAVOR DE LAS PYMES}

\subsection{La Comisión Europea y las Activi- dades en favor de las PYMES}

La Comisión Europea viene elaborando informes sobre la coordinación de las actividades a favor de la PYMES de conformidad a la decisión del Consejo del 9/12/1996 relativa la Tercer Programa Plurianual a favor de las Pequeñas y Medianas Empresas (PYMES) de la Unión Europea (1997-2000). Informes que forman parte del seguimiento de las Resoluciones del Consejo de 1996 sobre la coordinación y sobre el Programa Integrado y de las Resoluciones correspondientes al Parlamento de 1997.

El último informe (cuarto en 1997), al ofrecer un panorama global de las actividades comunitarias de asistencia a las PYMES, concretó los objetivos del Programa Integrado, concebido como marco para la coordinación de las actividades a favor de las PYMES. Este informe se basó, fundamentalmente, en dos prioridades: la mejora del entorno de las PYMES y los programas y medidas en su apoyo, y actualiza el último informe de 1995. Las conclusiones revelaron los progresos alcanzados desde la elaboración del primer informe de 1992.

El objetivo de la Comisión es conseguir que la dimensión PYMES se integre en la definición y aplicación de las políticas comunitarias e incrementar la participación de las PYMES en los Programas Comunitarios.

La aparición de una prioridad política a favor de las PYMES se ha ido desa- 
rrollando progresivamente en el seno de la Unión debido al reconocimiento del potencial de creación de puestos de trabajo de estas pequeñas y medianas instituciones, lo cual ha impactado positivamente en los últimos años en los Consejos Europeos.

Por otro lado, para mejorar el entorno administrativo, legal, fiscal y financiero de las PYMES, la Comisión está trabajando para tener en cuenta la especialidad de las PYMES en la elaboración de todas las políticas comunitarias (programa mercado interior, competencia, fiscalidad, medio ambiente y política social) y ha propuesto acciones específicas. La nueva definición de la PYME constituye una mejor orientación de las medidas a favor de estas empresas.

Para las legislaciones existentes, se están adoptando iniciativas para simplificar la legislación relativa al mercado interior. Luego del Consejo Europeo de Ámsterdam de junio de 1997, la Comisión ha creado mecanismos para mejorar y simplificar el entorno de las empresas, sobre todo las que se encuentran en fase de lanzamiento y vienen trabajando sobre los plazos de los pagos y la transmisión de empresas.

Los programas y medidas de apoyo a las empresas hacen referencia prioritariamente al acceso, al financiamiento y al crédito, el apoyo al desarrollo de las PYMES en regiones desfavorecidas, la europeización e internacionalización de las PYMES, el fortalecimiento de la competitividad, el mejor acceso a la investigación, la innovación, la formación y consideraciones medioambientales.

Las medidas de apoyo llevadas a cabo se refieren principalmente a la información mediante la red de los "Euro Info Centres", primeras ventanillas de acceso a la información comunitaria de las empresas. La europeización e internacionalización de las empresas a través de las redes de cooperación interempresarial (BC-NET y BRE) y los programas EUROPARTEN'ARIAT e INTERPRISE. Así como las acciones de subcontratación transnacional como la organización de salones de subcontratistas (International Buyers Exhibition - IBEX). Las actividades hacen referencia también a la artesanía, el comercio, que ha sido objeto de un libro verde, y la economía social.

Con el objeto de ayudar a las empresas artesanales y a las pequeñas empresas, se han realizado una serie de actuaciones para fomentar la cultura y el espíritu empresarial, y para apoyar a las mujeres empresarias. Para favorecer la creación de empresas conjuntas dentro de la Unión, la Comisión ha adoptado el nuevo programa denominado "JEV - Joint European Venture", también se ha puesto en marcha una acción Eurmanagement Environnement para ayudar a las PYMES a participar en el sistema comunitario de gestión medioambiental y de auditoría.

Los demás programas y medidas de apoyo comunitario que afectan a las PYMES actúan principalmente a través de los fondos estructurales, los programas de investigación, desarrollo tecnológico, de formación profesional y de cooperación internacional. Otras acciones afectan a las PYMES en los campos de la calidad y la normalización, la innovación, el medio ambiente(ECO- AUDIT), los transportes, la energía y la industria audiovisual.

Por otro lado, la participación de estas empresas en los programas se ha ido incrementando progresivamente. En los fondos estructurales, principales instrumentos financieros de la Unión, las PYMES son receptoras de las ayudas.

Entre los períodos 1989 - 1993 y 1994 -1999 , se puede observar casi una duplicación de las donaciones comunitarias. 
La Comisión también ha propuesto que el margen disponible de los programas existentes se utilice en provecho de las PYMES.

Por su parte el Banco Europeo de Inversiones ${ }^{5}$ ha duplicado el volumen de sus préstamos globales en favor de competitividad de estas entidades entre 1993 y 1996. El número de empresas beneficiarias también se ha duplicado hasta llegar a 11,034 en 1996. El fondo Europeo de Inversiones destina ahora el $34 \%$ de sus actividades a estas instituciones.

En los programas de Investigación y Desarrollo Tecnológico (IDT), la introducción de medidas específicas han permitido que se incremente la participación de éstas en el Cuarto Programa Marco, doblando casi su participación en relación con el Tercero. Además, en el marco del Programa de Innovación, la comisión puso en marcha, en julio de 1997, el proyecto piloto I-TEC para fomentar las inversiones en capital riesgo para PYMES tecnológicamente innovadoras.

Para favorecer la internacionalización, las metodologías e instrumentos comunitarios de información y cooperación existentes se han ampliado una serie de terceros países con el contexto de programas comunitarios, en el Mediterráneo (MEDA), en América Latina (AL-INVEST) y más recientemente en Asia (ASIA-INVEST), así como, en los países de Europa Central y Oriental (PHARE y COOPME) y en los países beneficiarios del programa TACIS.

En materia de formación profesional, as PYMES son una prioridad en los programas que dependen del objetivo 4 del Fondo Social Europeo y de la iniciativa
ADAPT cuyas dotaciones oscilan entre $50 \mathrm{y}$ $80 \%$, según los Estados miembros.

\subsection{Actividades de la Comunidad de Madrid y las Cámaras de Comercio a favor de las PYMES}

La comunidad de Madrid y las Cámaras de Comercio e Industria a lo largo de éstos 3 últimos años han adecuado áreas y/o oficinas específicas de Euro para atender las dudas y consultas de empresarios y comerciales y dar el asesoramiento respectivo.

Asimismo, han efectuado jornadas de exposición y debate de los efectos de Euro que sirven para la detección de problemas y dificultades en busca de una solución adecuada. Junto con el asesoramiento han organizado planes de formación específicos de recursos humanos para que estas empresas enfrenten los nuevos retos que va a suponer la introducción del Euro y la sustitución de la peseta.

Por su parte, el Gobierno en curso ha realizado una serie de medidas en apoyo a las PYMES a lo largo de sus 4 años de mandato. En el tema de la fiscalidad, redujo la carga fiscal, simplificó los trámites con la administración tributaria y ha facilitado la transmisión de empresas familiares. Respecto al tema de financiación mejoró la regulación de acceso de las PYMES al crédito y destinó alrededor de 1200,00 millones de pesetas en préstamo a las PYMES a través de la línea ICO.

Por otro lado, desarrolló un Plan de Agilización Normativa para la competitividad de la PYME; así como, han puesto en marcha 4 proyectos de Ventanillas Únicas Empresariales. 
En relación al tema de la internacionalización de las PYMES, el Gobierno creó FONDPYME, fondo para el fomento de la inversión de las PYMES españolas en el exterior. Además, creó la "póliza 100" que asegura las operaciones de exportación de estas entidades y se desarrolló un programa PIPE 2000 que ha conseguido 2000 nuevas PYMES exportadoras entre 1997 y 1999.

\section{CONCLUSIONES Y REFLEXIONES}

A modo de conclusión, podemos decir que con la moneda única desaparecerán los riesgos del tipo de cambio, lo que influirá en la reducción de los tipos de interés, impulsando el comercio y a la inversión. Los costes de la transacción de cambio durante las operaciones comerciales y algunos costes de gestión tenderán a desaparecer, obteniéndose una mayor rentabilidad de las inversiones.

La principal desventaja de la implementación de la moneda única serán los costes temporales que se generarán por el cambio que son el establecimiento de nuevos precios, la adaptación de tecnologías y la adaptación de sistemas.

Por otro lado, según nuestro análisis del entorno macroeconómico y microeconómico de las PYMES sugerimos que estas entidades diagnostiquen el ámbito de las áreas de la organización que potencialmente serán afectadas, con identificación de oportunidades y problemas y previsión de plazos.

Una valoración de los planes de acción necesarios para adaptarse al proceso, con establecimiento del calendario de actividades, prioridad de las tareas, recursos materiales y humanos a dedicar, plan de financiación de los mismos y determinación de responsabilidades y puntos de revisión y control.
El arranque y puesta en marcha inmediata de aquellas acciones que se consideren ineludibles o prioritarias bajo el seguimiento directo de algún miembro de la dirección que supervise el progreso, controle la desviación y determine las modificaciones a adoptar en el tiempo, en función de las circunstancias concurrentes, internas y externas, algunas de ellas aún pendientes de definición.

Consideramos que un enfoque de este tipo es clave hoy en día. Si la propia organización considera que no tiene medios para acometer con garantía de éxito la tarea, se deberá acudir a un personal externo especializado procedente de instituciones oficiales o de entidades privadas. En este punto creemos que las PYMES tendrán que apoyarse en las cámaras de comercio y en las asesorías de la comunidad.

Pensamos que la supervivencia de las PYMES -sobre todo las industriales-, va a depender en estos próximos años de su capacidad para potenciar sus recursos materiales e intangibles, desarrollar sus competencias esenciales en los campos organizativos y gestionar la innovación y comercialización. La innovación configurará el núcleo del espíritu empresarial.

El papel del sector público español respecto a estas empresas deberá centrarse en fomentar la innovación, creando un entorno favorable, orientando las investigación hacia el mercado, mejorando la formación permanente y apoyando la financiación.

El futuro de las PYMES, sobre todo de aquellas que se actúan dentro de los sectores de elevada tecnología, dependerá - a nuestro criterio-, de la existencia y articulación combinada de una política orientada al crecimiento, una política macroeconómica de estabilidad y de una política de reformas 
estructurales. Sólo la conjunción de las tres posibilita un crecimiento estable y la mejora de los factores productivos.

\section{BIBLIOGRAFIA}

AMIÁN MARTÍNEZ, Rafael

1998. "Posibles ventajas generales para las PYMES. Problemas o costes y grado de apertura al exterior según sectores". En: El euro y las empresas españolas. Cámara Oficial de Comercio e Industria de Madrid.

\section{BANCODE ESPAÑA}

1998. Central de balances: Resultados anuales de las empresas no financieras.

\section{BANCODE ESPAÑA}

1998. Central de balances: Registros mercantiles.

\section{BANCO DE ESPAÑA}

Febrero 2000. Informes Estadísticos. En: xeb http://www.bde.es/infoest/be0201.txt

\section{COMISIÓN EUROPEA}

1998. Actividades a favor de las PYMES y la industria.

En:web http://europa.eu.int/comm/g23/ een-policy/eu-act.SME/ com_1997_610_es.pdf

\section{COMISIÓN EUROPEA}

La unión monetaria y las PYMES.

En: web http://www cajasegovia.es/observatorio/europa/Pymes/temal.htm

DE LA VEGA, Ignacio

1998."La comunidad de Madrid y la adapación al euro de las PYMES". En: El impac- de la moneda única en las empresas inañolas. Cámara Oficial de Comercio e trdustria de Madrid.
GONZÁLES MINGUEZ, José/SANTILLÁN, Javier

Mayo 1998. "El papel del euro en el sistema monetario internacional y sus implicaciones para España". En: Boletín Económico del Banco de España, Madrid.

MINISTERIO DE ECONOMIA Y HACIENDA Enero 2000. Informe de Coyuntura Económica. Madrid.

MOLERO, José

1998. "Una ocasión para reorganizar la estrategia de las PYMES". En: El euro y las empresas españolas. Cámara Oficial de Comercio e Industria de Madrid.

SÁNCHEZ LANGEBER, Rafael

El euro y la empresa.

En: web http://www.geocities.com/ WallStreet/8999/europymes, html.

SANTANIELLO, Roberto

1999. El mercado único europeo. Editorial Acento. Madrid.

SERRA PERIS, José Manuel.

1996. "La PYME industrial en el nuevo modelo de crecimiento". En: Economía industrial. No. 310, Madrid.

TAMANÉS, Ramón

1998. Unión monetaria y el euro: La recta final. Editorial Espasa Calpe S.A.

Madrid.

TESOROPÚBLICO

2000. Boletín Mensual N ${ }^{\circ} 67$.

En: web http://www.meh.es/tsoro/deudo/ boletin/sp/apartados/bol.l.htm 